\title{
Contamination of Environment by Device
}

National Cancer Institute

\section{Source}

National Cancer Institute. Contamination of Environment by Device. NCI Thesaurus.

Code C139492.

Operation of the device results in contamination of the nearby environment e.g. dust, dirt, smoke, heat or biological material. 